\title{
Erosive Oral Lichen Planus and its Management: A Case Series
}

\author{
Sharma S, 'Saimbi CS, ${ }^{1}$ Koirala B' \\ B.P. Koirala Institute of Health Sciences, Dharan, Nepal
}

\section{ABSTRACT}

Lichen planus (LP), although a dermatosis, is more common in the oral mucous membrane than in the skin. Lesions of oral LP are classically found on the buccal mucosa and gingiva. Among the various types, the reticular lesions are asymptomatic and require no treatment, but pain and severe discomfort accompany the erosive or ulcerative lesions. Malignant transformation to squamous cell carcinoma developing in areas of erosive oral LP (EOLP) being a possibility, it is important for clinicians to maintain a high index of suspicion for all intraoral lichenoid lesions. Therefore, periodic follow-up of all patients with EOLP is recommended. In view of the above, this paper highlights the management of four cases of EOLP with topical corticosteroid and $\mathrm{CO}_{2}$ laser surgery.

Key words: carbon dioxide laser, corticosteroid, lichen planus

\section{INTRODUCTION}

Lichen planus (LP) is an autoimmune, chronic, inflammatory disease that affects mucosal and cutaneous tissues. Oral lichen planus (OLP) occurs more frequently than the cutaneous form and tends to be more persistent and more resistant to treatment. ${ }^{1}$ Although there are several clinical forms of OLP (reticular, patch, erosive, and bullous), the most common are the reticular and erosive forms. ${ }^{1}$

The exact etiology of LP is not known, but it is believed to result from an abnormal T-cell mediated immune response in which basal epithelial cells are recognized as foreign because of changes in the antigenicity of their cell surface. ${ }^{2}$ However, the cause of this immunemediated basal cell damage is still not known.
The erosive form is the second most common type of OLP. ${ }^{3}$ Atrophic and bullous forms are considered to be the variants of the erosive form. It presents as a mix of erythematous and ulcerated areas surrounded by finely radiating keratotic striae. Patients with erosive oral lichen planus (EOLP) present with symptoms ranging from episodic pain to severe discomfort. It is important for all clinicians to be aware of its clinical presentations and management since LP is one of the most common mucosal conditions affecting the oral cavity.

The most widely accepted treatment for OLP is topical corticosteroids. Alternative treatments include retinoids, cyclosporine, tacrolimus, surgery and cabon dioxide $\left(\mathrm{CO}_{2}\right)$ laser. This paper highlights the use of topical corticosteroid and $\mathrm{CO}_{2}$ laser for the management of four cases of EOLP.

Correspondence:

Dr. Shivalal Sharma

Department of Periodontics

B.P. Koirala Institute of Health Sciences, Dharan, Nepal

Email: drshivalalsharma@yahoo.co.uk 
All the patients attended the Postgraduate Clinics of Periodontics, Faculty of Dental Sciences, KG's University of Medical Sciences, Lucknow, India. At the base line visit, digital photographs of the lesions were taken and full-mouth ultrasonic scaling carried out. Thereafter, incisional biopsy of the affected area was obtained under local anesthesia and sent to the Department of Pathology for histopathological examination. Patients were recalled with the biopsy report. This was the common protocol followed for all the four cases.

\section{FIRST CASE}

A 45-year-old lady reported with the chief complaint of burning sensation of gums for the last 15 days while taking hot and spicy foods. Intraoral examination revealed erythematous, desquamated lesions circumscribed by fine keratotic striae involving both the marginal and attached gingiva of the mandibular and maxillary anterior teeth labially (Figure 1a). There were no significant local deposits on the corresponding teeth. No cutaneous lesions were visible. The patient's past medical, dental, and family histories were not significant. Histopathological findings revealed hyperkeratosis, hydropic degeneration of the basal layer and a dense band like infiltrate of T-lymphocyte in the lamina propria. Based on the clinical and histopathological findings, final diagnosis of lichen planus was made.

The patient was then instructed to apply a thin layer of triamcinolone acetonide $(0.1 \%)$ ointment directly on the lesion three times a day (after meals and at bedtime), and not to take anything orally for one hour after its application, following which she was to rinse with Chlorhexidine $(0.2 \%)$ mouthwash. On the twoweek-recall visit, though less, burning sensation and erythematous areas were still persistent. Oral hygiene instructions were reinforced and the patient was asked to continue the same medication and recalled again after one month. However, the patient reported after six weeks and on re-evaluation, the erythematous desquamated area and burning sensation were absent. The generalized Chlorhexidine staining of teeth present removed with ultrasonic scaling and polishing (Figure1b).

\section{SECOND CASE}

A 35-year-old lady reported with the chief complaint of severe discomfort in the upper left side of the jaw since three weeks. Clinical examination revealed fine, white, radiating striations bordering an erosive area involving the upper left buccal gingiva and alveolar mucosa. Oral hygiene status was good. The patient's personal and medical histories were not significant. There was no corresponding skin lesion. Histopathological examination confirmed LP. The patient was prescribed triamcinolone acetonide $(0.1 \%)$ ointment followed by Chlorhexidine mouthwash with the same instructions as mentioned in the first case.

Follow-up done in 15 days showed improvement in the signs and symptoms of the lesion; however, the patient was still not totally symptom-free. Questioning revealed poor patient compliance with frequent missed application of ointment. A second recall visit after a month found the patient totally symptom-free with no intra-oral lesions.

\section{THIRD CASE}

A 48-year-old lady reported with the chief complaint of burning sensation of the mouth since one month. While examining the patient's oral cavity, red erosion of gingiva and alveolar mucosa was seen involving the mandibular premolar region. Local deposits were inconsistent with the corresponding lesion. No skin lesion was visible. Medical and dental histories were non-contributory.

Histopathological examination confirmed the diagnosis of LP and the lesion was treated with $\mathrm{CO}_{2}$ laser. Ablation of the erythematous mucosa was accomplished without any bleeding, charring or carbonization. Nimesulide 100 mg was prescribed for analgesia. Healing was complete in two weeks of follow-up with complete resolution of erythematous area and cessation of the burning sensation. No infections or any other postoperative complications were encountered.

\section{FOURTH CASE}

A 22-year-old boy reported with the chief complaints of reddish gums and discomfort while brushing in the lower back teeth since a week. Clinical examination showed red desquamated gingiva in relation to the mandibular first molar area buccally and white reticular lesions on the vestibular and buccal mucosa of the same side. Extra-oral examination revealed no relevant findings. Past medical and dental histories were not significant.

Based on the histopathological findings, the lesion was diagnosed as EOLP. The erythematous desquamated lesions were then ablated by $\mathrm{CO}_{2}$ laser in the superpulse mode under local anesthesia. $\mathrm{CO}_{2}$ surgical laser unit (Luxar NOVA Pulse Lx-20 SP, Bothwell, Wash) with a hollow fiber delivery system and noncontact air cooling handpiece was used under standard protective measures. Noncontact focused beam at repeated superpulse mode $(10$ watts, $0.8 \mathrm{~mm}$ spot size, $20 \mathrm{~Hz}$, 10 milliseconds) was applied in a circular motion and the remnants of the ablated tissue were removed using sterile gauze dampened with saline. This procedure was repeated until the desired depth of tissue removal was achieved (Figure 2a). The adjacent teeth were 
protected from the laser beam by application of an acrylic template covering the labial surface of the teeth, by using a smaller laser tip with a spot size of $0.4 \mathrm{~mm}$ at the gingival margins and interdental papilla, and powersetting reduced to 5 watts. Nimesulide $100 \mathrm{mg}$ was prescribed to be taken as needed. Slight discomfort was noted by the patient only on the first two days after the treatment. On the two-week follow-ups, the healing of the laser-treated wound was found to be complete and uneventful. Burning sensation and the erythematous areas were not present (Figure $2 b$ ).

\section{DISCUSSION}

Lichen planus is a relatively common disorder that is estimated to affect $0.5 \%$ to $2.0 \%$ of the general population. ${ }^{4}$ LP affects primarily middle-aged adults, and the prevalence is greater among women. ${ }^{5}$ Children are rarely affected. ${ }^{6}$ The classic skin lesions of the cutaneous form of LP can be described as purplish, polygonal, planar, pruritic papules and plaques. ${ }^{7}$ These skin lesions commonly involve the flexor surfaces of the legs and arms, especially the wrists. The nail beds may also be affected with resultant ridging, thinning and subungual hyperkeratosis. ${ }^{7}$ Scalp involvement if untreated can lead to scarring and permanent hair loss. In a dental setting, cutaneous LP is observed in about one third of the patients diagnosed with OLP. ${ }^{8}$ In contrast, two-third of patients seen in dermatologic clinics exhibit OLP. ${ }^{9}$ Up to $10 \%$ of patients with OLP have lesions restricted to the gingival tissue. ${ }^{9}$ Although the oral cavity may present LP lesions with a distinct clinical configuration and distribution, the clinical presentation sometimes may simulate other mucocutaneous disorders. ${ }^{10}$ Therefore, for the final diagnosis to be made the clinical diagnosis of OLP should always be accompanied by histopathologic or immunologic diagnosis.

Some studies indicate an increased risk of squamous cell carcinoma in patients with OLP lesions. ${ }^{11,12}$ This increased risk appears most common with the erosive form of OLP. A review of previously published studies concluded that the risk of developing squamous cell carcinoma in patients with OLP is approximately ten times higher than that in the unaffected general population. ${ }^{13}$ Therefore, clinicians should maintain a high index of suspicion for intra-oral areas that appear unusual even in patients with histologically confirmed diagnosis of EOLP.

Currently, there is no cure for OLP. Excellent oral hygiene is believed to reduce the severity of the symptoms, but it can be difficult for patients to achieve high levels of hygiene during periods of active disease. Treatment is aimed primarily at reducing the length and severity of symptomatic outbreaks. Asymptomatic reticular and plaque forms of OLP do not require pharmacologic intervention.

Topical corticosteroids are the mainstay in treating mild to moderately symptomatic lesions. Options (presented in terms of decreasing potency) include $0.05 \%$ betamethasone valerate gel, $0.05 \%$ fluocinonide gel, and $0.1 \%$ triamcinolone acetonide ointment. ${ }^{6,14}$

The use of topical steroids has fewer side effects than systemic administration. The disadvantages include: candidiasis, thinning of the oral mucosa, compliance problem, and discomfort while applying. Topical formulations of the more potent corticosteroids can cause adrenal suppression if used in large amounts for prolonged periods. The lowest-potency steroid that proves effective should be used. Steroids use is contraindicated in patients who are breast feeding, and used with caution in patients with herpetic infections, glaucoma, pregnancy, HIV infection, tuberculosis, diabetes mellitus, candidiasis, and hypertension. The prophylactic use of a $0.2 \%$ Chlorhexidine gluconate rinse may help reduce the incidence of fungal infection during corticosteroid therapy. ${ }^{15}$

$\mathrm{CO}_{2}$ laser has been used for a variety of oral surgical procedures: gingivectomy, gingivoplasty, frenectomy, incisional and excisional biopsy, and operculectomy. ${ }^{16}$ $\mathrm{CO}_{2}$ laser surgery of the oral tissues is generally performed with a power setting of five to fifteen watts in either a pulsed or continuous mode. ${ }^{16}$ Another mode of pulse duration in $\mathrm{CO}_{2}$ laser system is the superpulse mode, which release bursts of higher peak powers and shorter pulse duration in the microsecond range. This mode allows the surgeon to deposit a pulse of higher peak power into the tissue with control, confine the exposures to pulses that are within thermal relaxation time of the tissue, and use pulse repetition rates that allow tissue cooling between individual pulses to reduce heat accumulation in tissue. ${ }^{17}$ Local anesthesia was used to control pain which otherwise could have hindered complete ablation of the lesion. $\mathrm{CO}_{2}$ laser was used cautiously and the adjacent tooth protected, since inappropriate application has shown to cause gingival recession, damage to the underlying periosteum and bone, delayed wound healing, and loss of enamel. ${ }^{18}$

The depth of thermal damage for the $\mathrm{CO}_{2}$ laser extends from 50 to $100 \mu \mathrm{m}$ compared to $200 \mu \mathrm{m}$ for Argon and $600 \mu \mathrm{m}$ for the $\mathrm{Nd}$ : YAG laser. ${ }^{19}$ Therefore, one can expect a lower risk with $\mathrm{CO}_{2}$ laser to the periosteum and the underlying bone. The results of the present study also support this idea, the $\mathrm{CO}_{2}$ laser use producing no periosteal damage or gingival fenestration.

Carbonization and charring are untoward effects of continuous wave of $\mathrm{CO}_{2}$ laser. Charring occurs when 


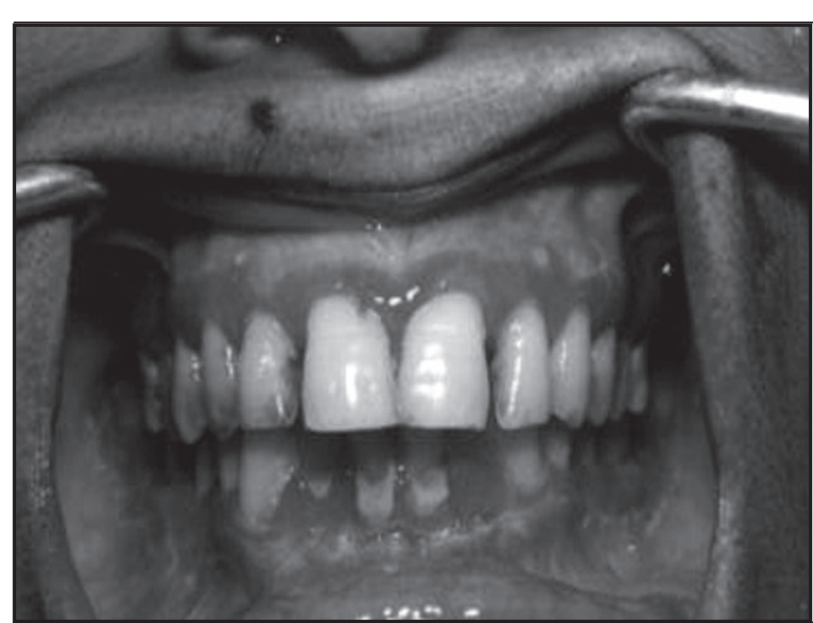

Figure 1a. Erythematous, desquamative lesion restricted to the gingiva

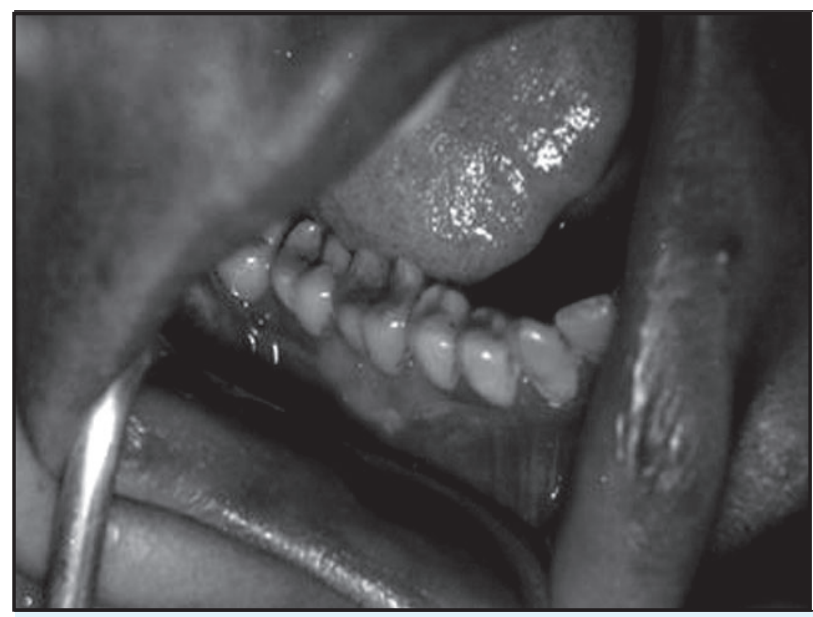

Figure 2a. Immediately after CO2 laser surgery

the tissue absorbs heat faster than it can release it via conduction or circulation. In the superpulse mode, each pulse has a higher energy and is of very short duration (less than $800 \mu \mathrm{s}$ ) thereby minimizing unwanted thermal damage to the surrounding tissues. A high power setting with short and low frequency pulses (10 watts, 10 milliseconds, $20 \mathrm{~Hz}$ ) was used in our cases in order to achieve adequate and controlled tissue removal which reduced overall operative time.

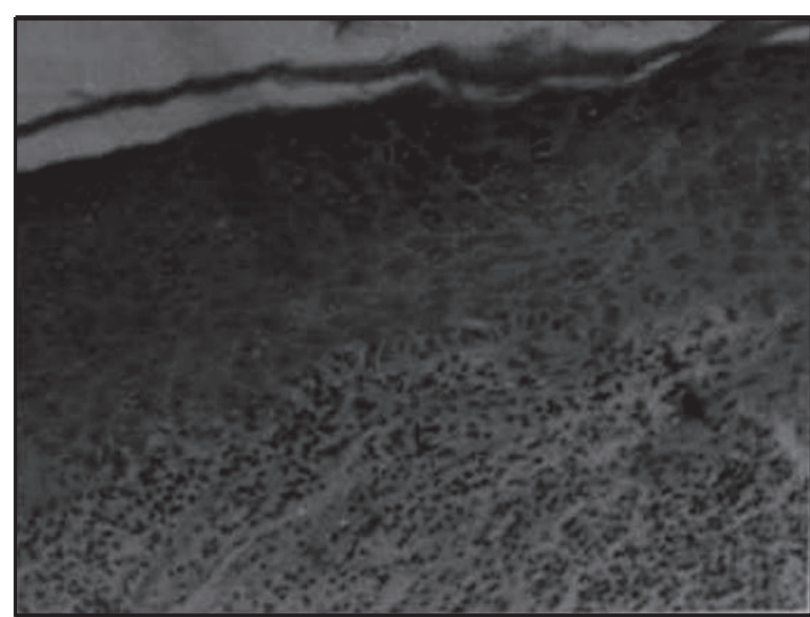

Figure 1b. After six weeks of topical corticosteroid treatment, and after scaling and polishing

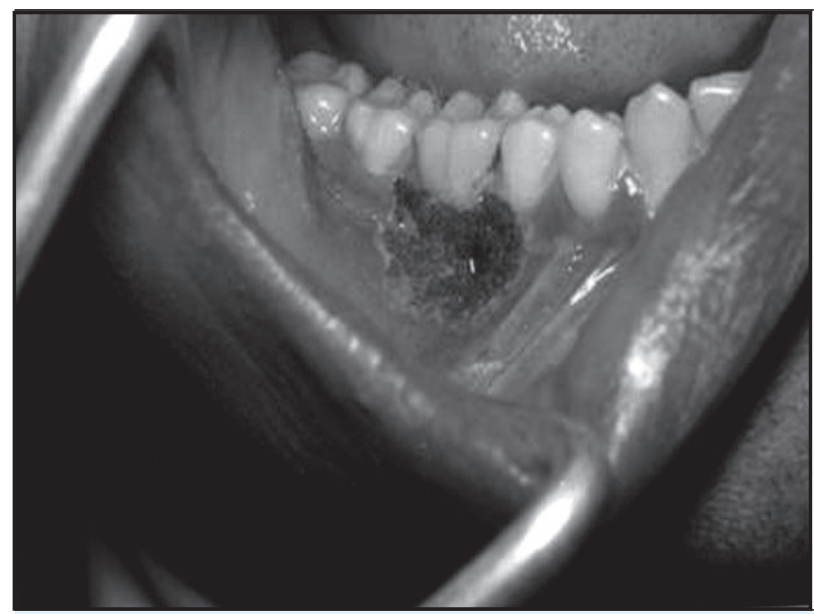

Figure 2b. After two weeks of $\mathrm{CO} 2$ laser surgery

\section{CONCLUSION}

Topical corticosteroids constitute the mainstay of treatment for symptomatic lesions of EOLP. It is effective, easily available and affordable, but has compliance problem because of discomfort, and frequent applications. The use of $\mathrm{CO}_{2}$ laser appears to be safe and effective alternative procedure for the treatment of EOLP; however its use is limited as it is quite expensive.

\section{REFERENCES}

1. Mollaoglu N. Oral lichen planus: a review. Brit J Oral Maxillofacial Surg 2000;38(4):370-7.

2. Sapp JP, Eversole LR, Wysocki GP. Contemporary Oral and Maxillofacial pathology; St. Louis (MI): Mosby; 1997.

3. Edwards PC, Kelsch R. Oral lichen planus: Clinical presentation and management. J Can Dent Assoc 2002;68(8): 494-9.
4. Mc Creary CE, Mc cartan BE. Clinical management of lichen planus. Brit J Oral Maxillofacial surg 1999;37(5):338-43.

5. Brown RS, Bottomley WK, Puente E, Lavigne GL. A retrospective evaluation of 193 patients with oral lichen planus. J Oral Pathol Med 1993;22(5):69-72.

6. Jungell P. Oral lichen planus: a review. Int J Oral Maxillofac Surg 1991;20(3):129-35. 
7. Katta R. Lichen Planus. Am Fam Physician 2000 Jun 1; 61(11)3319-24,3327-8.

8. Lozoda-Nur F, Miranda C. Oral lichen planus: Epidemiology, clinical characteristics, and associated diseases. Sem Cutan Med Surg 1997;16: 273.

9. Scully C, El-kom M. Lichen planus: Review and update on pathogenesis. J Oral Pathol 1985;14:431.

10. Regezzi JA, Sciubba JJ. Oral pathology: Clinical pathologic correlations, 3rd ed. Philadelphia: WB Saunders; 1999.

11. Barnard NA, Scully C, Eveson JW, Cunningham S, Porter SR. Oral cancer development in patients with oral lichen planus. J Oral Pathol Med 1993;22(9):421 - 4.

12. Silverman S: Oral lichen planus. a potiantially premalignant lesion. J Oral Maxillofacial Surg 2000;58(1):1286-8.

13. Drangsholt M, Truelone EL, Morton TH Jr, Epstein JB. A man with a thirty-year history of oral lesions. J Evid Base Dent Pract 2001;1(2):123-35.

14. Vincent SD. Diagnosing and managing oral lichen planus. JADA 1991;122(6):93-96.
15. Ellepola AN, Samaranayeke LP. Adjunctive use of Chlorhexidine in oral candidoses: a review. Oral Dis 2001;7(1):11-17.

16. Position paper of American Academy of Periodontology: Lasers in Periodontics. J Periodontol 2002;73:1231-9.

17. Hobbs ER, Bailin PL, Wheeland RG, Ratz JL. Superpulsed Lasers: Minimizing thermal damage with short duration, high irradiance pulses. J Dermatol Surg Oncol 1987;13:95564 .

18. Miserendino LJ, Pick RM, Blankenau RJ. Laser safety in dental practice. In: Miserendino LJ, Pick RM, editors. Lasers in Dentistry. Chicago, Ill: Quintessence Pub. $\mathrm{CO}_{2} ; 1995$ P.85 $-102$.

19. Esen E, Haytac MC, Oz IA, Erdogan O, Karsli ED. Gingival melanin pigmentation and its treatment with the $\mathrm{Co}_{2}$ laser. Oral Surg Oral Med Oral Pathol Oral Radiol Endod 2004; 98(5):522-7. 